Mirai. Estudios Japoneses

ISSN-e: 1988-2378

http://dx.doi.org/10.5209/MIRA.57103

\title{
Arquitectos viajeros: influencias de ida y vuelta en el Japón de entreguerras
}

\author{
$\mathrm{D}^{\mathrm{a}}$ Lilian Barranco Luque ${ }^{1}$
}

Resumen: Entre finales del siglo XIX y las primeras décadas del siglo pasado, la sociedad japonesa se encontraba experimentando un cambio cultural de gran envergadura, su mirada se volvió hacia el mundo occidental y abrazó con entusiasmo no sólo los aspectos tecnológicos y políticos procedentes de Occidente, sino también los modos y costumbres de ese nuevo referente exterior, una vez China quedó desplazada de ese papel. El desarrollo de los medios de transporte en la misma época facilitó el intercambio de experiencia en amplios ámbitos del conocimiento entre los dos mundos. Numerosos intelectuales y estudiantes viajaron a otros países con la intención de tener acceso directo a los grandes centros del saber en Occidente. Entre los profesionales y estudiosos que viajaban a Europa y Estados Unidos se encontraban también los arquitectos recién graduados en la joven Universidad Imperial de Tokio. El viaje se convirtió en un factor decisivo en la influencia de ida y vuelta que se produjo entre arquitectos occidentales y japoneses, especialmente durante el periodo de entreguerras. Los viajes a Occidente supusieron un gran salto para la arquitectura moderna en Japón tanto por el aprendizaje de técnicas y teorías novedosas, como por la toma de consciencia de los arquitectos japoneses de su propia particularidad y originalidad estética, ya que muchos de los rasgos fundamentales de la arquitectura japonesa fueron observados por estos arquitectos viajeros en el trabajo de algunos de sus colegas en Occidente.

Palabras claves: Arquitectura moderna japonesa; entreguerras; viaje.

\section{[en] The traveller-architect: mutual influences in inter-war Japan}

\begin{abstract}
Between the late $19^{\text {th }}$ century, and the early decades of the 20th, Japanese society underwent a major cultural change. It turned its gaze towards the Western world and enthusiastically embraced not only technological and political aspects but also the ways and customs of its new external reference, once China had been divested of that role. Meanwhile, the development of transport facilitated the exchange of experience in broad areas of knowledge between the two worlds. Many intellectuals and students travelled to other countries with the intention of having direct access to the most important education centres in the Western world. Amongst professionals and scholars who travelled to Europe and the United States, there were graduate architects from the Imperial University of Tokyo. The trip became a decisive factor in the mutual influence that developed between Western and Japanese architects, especially during the interwar period. Traveling to the West became a major step forward for modern Japanese architecture, not only in learning new techniques and theories, but also in making Japanese architects conscious of their own singularity and aesthetic originality, as many of the fundamental features of Japanese architecture were observed by these traveller-architects in the work of some of their Western colleagues.
\end{abstract}

Keywords: Modern Japanese architecture; inter-war; travel.

Sumario. 1. Introducción. El viaje como fuente de conocimiento y desarrollo. 2. Influencias de ida y vuelta. 3. Conclusiones.

Cómo citar: Barranco Luque, L. (2017). Arquitectos viajeros: influencias de ida y vuelta en el Japón de entreguerras, en Mirai. Estudios Japoneses 1(2017), 83-92.

1 Universidad de Málaga.

lilian.barranco@gmail.com 
Para un hombre en la edad madura es un gran privilegio viajar alrededor del mundo y tener la oportunidad de comparar la actividad humana mientras intenta discriminar entre lo esencial y lo circunstancial.

Walter Gropius

\section{Introducción. El viaje como fuente de conocimiento y desarrollo}

Mientras que el turismo es de manera genérica un fenómeno fundamentalmente moderno, el viaje, entendido como vehículo para la adquisición de experiencia y fuente de conocimiento, ha formado parte de la historia del ser humano desde sus inicios y constituyendo un elemento esencial en el avance y enriquecimiento de las sociedades a través del comercio, la exploración, el intercambio e incluso la conquista.

Fueron las élites europeas del siglo XVIII las que comenzaron a viajar por primera vez persiguiendo objetivos alejados de los planteamientos expansionistas o mercantilistas, en lo que vino a llamarse el Grand Tour, a la Italia del momento con el único fin de conocer la cuna de la civilización occidental. Más adelante, Oriente se convierte en el destino soñado por los viajeros occidentales ávidos de conocimiento y aventura, siendo en este caso Turquía, Egipto o el Mediterráneo oriental y meridional, la traducción de esos anhelos de exotismo y sensualidad. Por fin, es a mediados del siglo XIX cuando el interés por el lejano Oriente crece de manera intensa en una Europa que descubre "lo japonés" y queda fascinada por el refinamiento de su arte y lo pintoresco de sus costumbres. A comienzos del siglo XX el desarrollo de los medios de transporte hace posible que los viajes entre estos dos mundos, Occidente y Japón, tan alejados en lo físico y lo cultural hasta ese momento, comiencen a producirse de manera regular.

Es en este contexto en el que la arquitectura japonesa de principios del siglo pasado se expande, se hace más compleja, se desarrolla y se universaliza. Tras la Primera Guerra Mundial, en el periodo que transcurre antes del segundo enfrentamiento global, la arquitectura japonesa se convierte, en palabras del profesor Oshima Ken, en "independiente de manera consciente, nunca más será una modernidad derivada o alternativa que simplemente siga los movimientos europeos o norteamericanos"3.

El viaje como actividad casi obligatoria para el desarrollo profesional y personal del individuo moderno se convierte en un factor decisivo en la mutua influencia e intercambio de conocimiento entre arquitectos occidentales y japoneses. Este interés por conocer al otro es consecuencia de la apertura que progresivamente fue experimentando la sociedad japonesa en general, y el mundo del arte y la arquitectura en particular, y que lleva a arquitectos abanderados del Movimiento Moderno en Japón, como los miembros del grupo Bunriha, Horiguchi Sutemi, Ishimoto Kikuji o Yamada Mamoru, a discípulos de Le Corbusier como Maekawa Kunio y Sakakura Junzō o también al arquitecto y fotógrafo seguidor de la Bauhaus, Yamawaki Iwao, entre otros, a realizar viajes por Europa y Estados Unidos. Siguiendo pasajes de la

2 Gropius, Walter / Tange, Kenzo / Ishimoto, Yasuhiro (1960): Katsura. Tradition and creation in Japanese Architecture. New Havens: Yale University Press, p. 1. (Trad. de la autora.)

3 Oshima, Ken Tadashi (2010): International Architecture in Interwar Japan: constructing Kokusai Kenchiku. Seattle: University of Washington Press, p. 10. (Trad. de la autora.) 
biografía de algunos de estos arquitectos, teniendo en cuenta también a los autores occidentales que desarrollaron parte de su carrera en el país nipón, como Frank Lloyd Wright, Antonin Raymond o Bruno Taut, podemos establecer la importancia que esos viajes tuvieron tanto en el intercambio de experiencias, técnicas y teorías, como en la toma de consciencia de los arquitectos japoneses de su propia particularidad y originalidad estética.

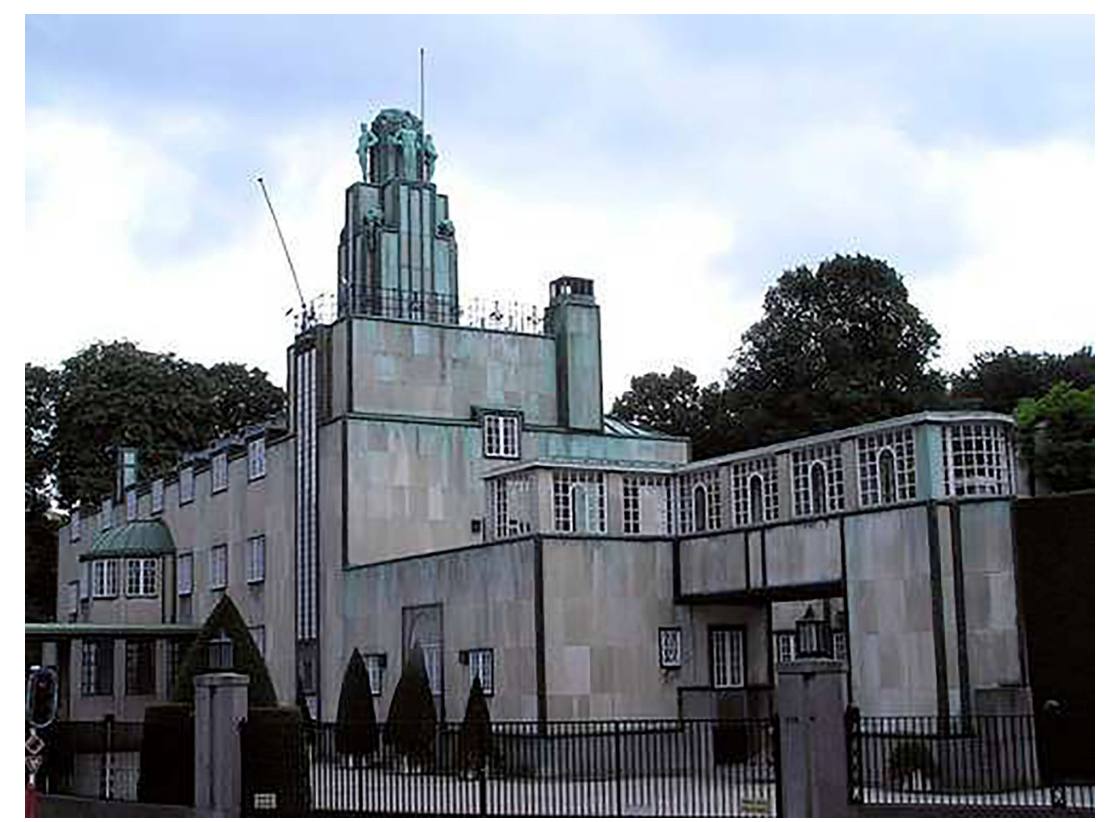

Fig. 1. Josef Hoffmann, Palacio Stoclet, 1905-1911. Tomada del sitio https://commons. wikimedia.org/wiki/File:Woluwe-St-Pierre_-_Hoffmann_050917_(1).jpg

\section{Influencias de ida y vuelta}

En 1868, año de la Restauración del gobierno imperial y comienzo del periodo Meiji, Japón da fin a un periodo de aislamiento de más de doscientos cincuenta años $\mathrm{y}$, desde el punto de vista de la cultura y las artes, un país que se había mostrado inaccesible durante tanto tiempo, se convierte en un verdadero descubrimiento para el mundo occidental. El creciente interés por Oriente, en concreto esta fascinación por lo japonés, dio lugar a la lo que denominamos japonismo, que surgido entre los círculos artísticos más vanguardistas de la Europa de finales del siglo XIX no dejó de ser una moda y como todas las modas terminó apagándose en ciudades como Londres o París. Sin embargo, siguió siendo acogido con profunda admiración en la Viena de principios del siglo XX, fenómeno éste del que fue testigo Horiguchi Sutemi en su viaje a Europa iniciado en julio de 1923. Los secesionistas vieneses, artistas y arquitectos, materializaron en sus obras esa admiración. En particular, Joseph Hoffman realizó en el Palacio Stoclet en Bruselas una suerte de "versión" de las 
casas de té japonesas [fig.1]. Esta obra es un ejemplo de la gesamtkunstwerk u obra de arte total, al integrar el trabajo de arquitectos, escultores, pintores y artesanos del grupo Wiener Werkstätte (Taller Vienés). A pesar de que el exterior está recubierto de mármol, un material prácticamente inexistente en la arquitectura tradicional japonesa, la visión de esta vivienda de lujo, construida para el industrial belga Adolph Stoclet, evoca las paredes y puertas compuestas por paneles de madera o de papel de arroz enmarcados en madera de las casas japonesas (fusuma y shōji). Más evidente aún resulta la influencia del arte japonés en el interior del palacio. La obra encargada para la decoración del comedor a Gustav Klimt, uno de los más ardientes admiradores del arte japonés entre los secesionistas, conocido como el Friso Stoclet, presenta claras reminiscencias de las figuras femeninas japonesas en el llamado Árbol de la Vida, especialmente en la figura conocida como La Expectación.

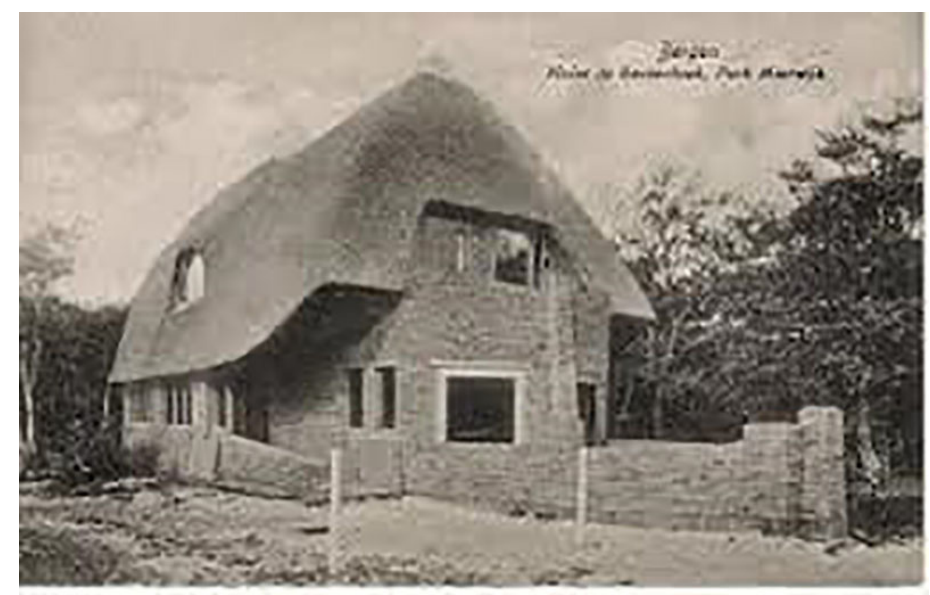

Fig. 2. Margit Kropholler, Villa Beukenhoek, 1916-1918. Tomada del https://commons.wikimedia.org/wiki/File:Villa_VI,_Meerwijklaan_7,_M.Kropholler_-_ Bergen_-_20031362__-_RCE.jpg

Asimismo, Horiguchi pudo también observar en la cultura y el diseño holandés el efecto del intercambio cultural de más de tres siglos entre los dos países. Los lazos entre Holanda y Japón se desarrollaron en torno a los vínculos comerciales a través del único puesto comercial occidental de Japón en la época Edo fundado por los holandeses en la isla de Hirado en 1609 y trasladado posteriormente al puerto de Nagasaki. El arquitecto japonés queda sorprendido por el amplio conocimiento que de Japón se tiene en Holanda en la época de su visita. En el ámbito de la arquitectura, concretamente de la arquitectura expresionista holandesa, el uso innovador de un material tradicional como la paja, cuyo mayor exponente es el diseño de la Villa Beukenhoek del Parque Meerwijk del arquitecto Margit Kropholler [fig. 2], una versión vernácula del estilo internacional, deja una profunda huella en Horiguchi como se verá pocos años después en la Villa Shiensō (1926). Las similitudes entre la Villa Beukenhoek y algunos tipos de arquitectura ancestral exenta de sofisticación como las tradicionales casas de tejados de paja características del medio rural en Japón debieron resultar muy reveladoras para Horiguchi [fig. 3]. 
Para Horiguchi, "Park Meerwijk significó la constatación de que el estilo de arquitectura japonesa sukiya, expresión de la arquitectura propia de las casas de té cuyo mejor ejemplo es la Villa Katsura (S. XVII), era realmente arquitectura moderna"4.

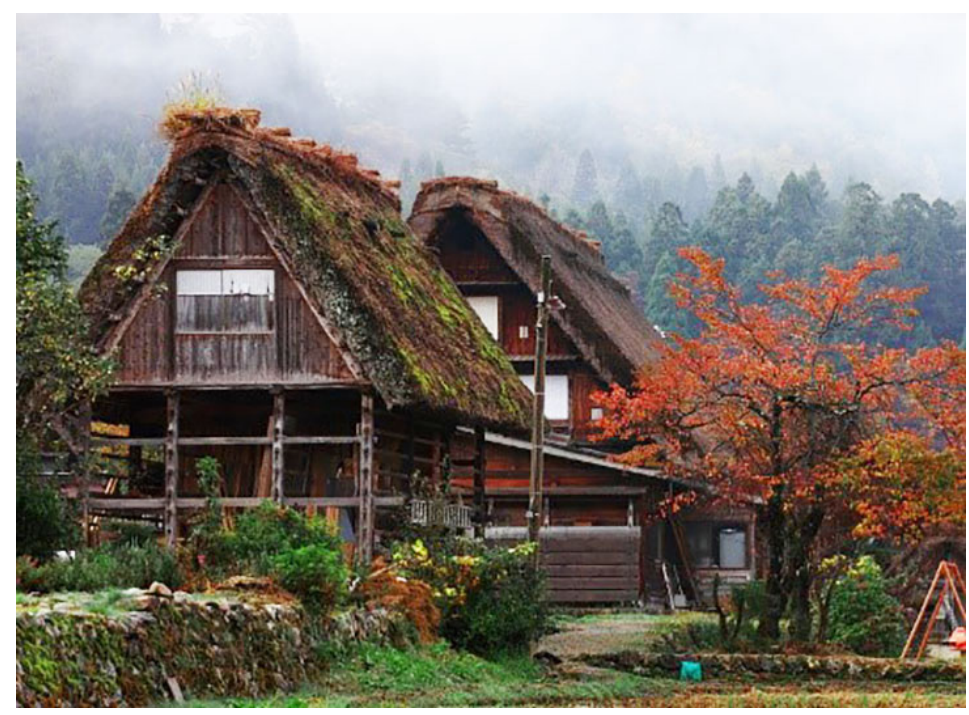

Fig. 3. Casas tradicionales japonesas. Tomada del sitio https://revolviendolavalija.wordpress.com/tag/japon-rural/

Por su parte, el también miembro del grupo Bunriha, Yamada Mamoru, fue enviado por el Ministerio de Comunicación a un viaje por más de diez países occidentales entre los años 1929-30. En un itinerario particularmente centrado en los países de habla germana, Yamada tuvo la oportunidad de tener numerosas entrevistas con figuras prominentes de la arquitectura moderna europea como Max y Bruno Taut, Walter Gropius y Erich Mendelsohn, que asimismo le dieron acceso a otros arquitectos como Le Corbusier, Mart Stam y J.J.P. Oud. Si para Horiguchi el encuentro con la arquitectura occidental le ayudó a redescubrir sus raíces vernáculas, para Yamada el contacto con Occidente y especialmente su participación en la segunda reunión del Congrès Internationaux d'Architecture Moderne (CIAM), que tuvo lugar en Frankfurt en 1929, provocó un profundo efecto en sus convicciones internacionalistas desde un punto de vista claramente social. Ambos aspectos, internacionalización y preocupación por lo social, son características que el profesor Oshima destaca en el caso de Yamada, facetas del Estilo Internacional que entendemos van íntimamente ligadas al concepto mismo de arquitectura moderna.

Entre los arquitectos viajeros japoneses queremos detenernos brevemente en el caso de Maekawa Kunio. Sin llegar a profundizar en la descripción del impacto que sus viajes a Occidente tuvieron en el desarrollo de su propio trabajo, la experiencia de Maekawa nos revela cómo el viaje no solo tiene efectos sobre la persona que se traslada en sentido literal, sino que también modifica el entorno estático que no se ha

4 Oshima, Ken Tadashi (2010): International..., op. cit., p. 59. (Trad. de la autora.) 
desplazado físicamente pero que queda influenciado y modificado por la filtración que provoca la experiencia viajera en sí misma. El 31 de marzo de 1928, la noche de su graduación en la Universidad Imperial de Tokio, Maekawa parte hacia París para trabajar en el estudio de Le Corbusier. La relación de este último con Japón cubre medio siglo de historia desde 1920 hasta 1960 y puede estudiarse desde varias perspectivas debido a la enorme relevancia de la figura del arquitecto suizo y su influencia en la arquitectura moderna, sin embargo, lo que aquí nos interesa destacar es cómo antes del viaje del propio Maekawa a la Europa de finales de los años 20, y a pesar de que existía todavía un conocimiento limitado del trabajo de Le Corbusier en Japón, Maekawa había quedado profundamente influenciado por los escritos de Le Corbusier tras su "gran viaje" a Grecia y Turquía, momento en el que el maestro europeo opta por rechazar cualquier tipo de decoración gratuita y abraza definitivamente los principios de la simplicidad, considerando que los logros del pasado quedan finalmente superados ${ }^{5}$.

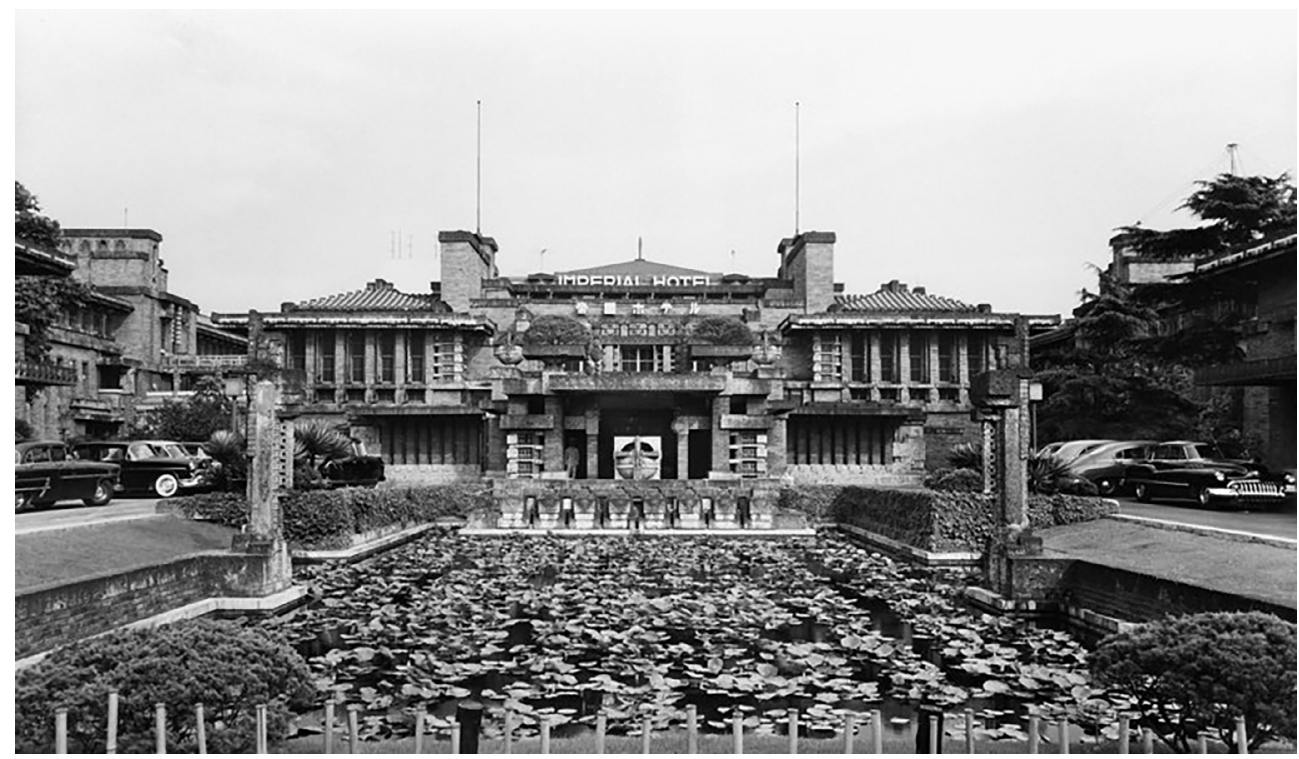

Fig. 4. Frank Lloyd Wright, Hotel Imperial de Tokio, 1916-22. Tomada del sitio https://commons.wikimedia.org/wiki/File:Imperial_Hotel_Wright_House.jpg

En cuanto al trabajo de arquitectos occidentales en Japón sobresale el caso de Frank Lloyd Wright, por su trascendencia en la historia de la arquitectura y la influencia que su presencia en el archipiélago nipón dejó entre los arquitectos de la generación nacida alrededor de 1890. Y esto a pesar de la opinión de Henry-Russell Hitchcock que encontramos en su Arquitectura: siglos XIX y XX, según la cual la

Reynolds, Jonathan M. (1991): Maekawa Kunio and the emergence of modernism in Japanese architecture. Ann Arbor, Michigan: UMI Dissertations Services. Reprint on the author's thesis (Ph.D.). Stanford University. 
obra de Wright en Japón supuso un "callejón sin salida" en la trayectoria del arquitecto nacido en Wisconsin. Su primer viaje de tres meses al país asiático tuvo lugar en 1905. Wright declaró que esta visita tenía como principal objetivo la compra de estampas japonesas, pero las fotos que realizó para documentar el viaje demuestran la profunda impresión que el paisaje y la arquitectura del país tuvieron sobre el arquitecto ${ }^{7}$. Años después Wright es invitado por la corte imperial japonesa a realizar el proyecto del Hotel Imperial de Tokio que se prolonga a lo largo de varios años 1916-1922. [fig. 4]. En las obras de este proyecto participaron varios arquitectos occidentales, entre ellos el checo-americano Antonin Raymond.

Según Oshima, la relación entre Wright, Raymond y Japón surge realmente a raíz del conocimiento que tiene este último de la interpretación de las estampas y de la estética japonesa del arquitecto norteamericano a través de traducciones de la obra Ausgefürhte Bauten und Entwürfe von Frank Lloyd Wright, publicada por Ernst Wasmuth en 1910. En 1916, Raymond se traslada a vivir a Taliesin, la residencia y estudio de Wright en Wisconsin. Las primeras impresiones sobre Japón fueron moldeadas por la visión del propio Wright y su entusiasta y romántica descripción del país asiático. En 1919 el maestro propuso al pupilo como ayudante en las obras del Hotel Imperial de Tokio, un hecho que en palabras del propio Raymond supusieron "el más maravilloso golpe de suerte" ${ }^{8}$ de su vida.

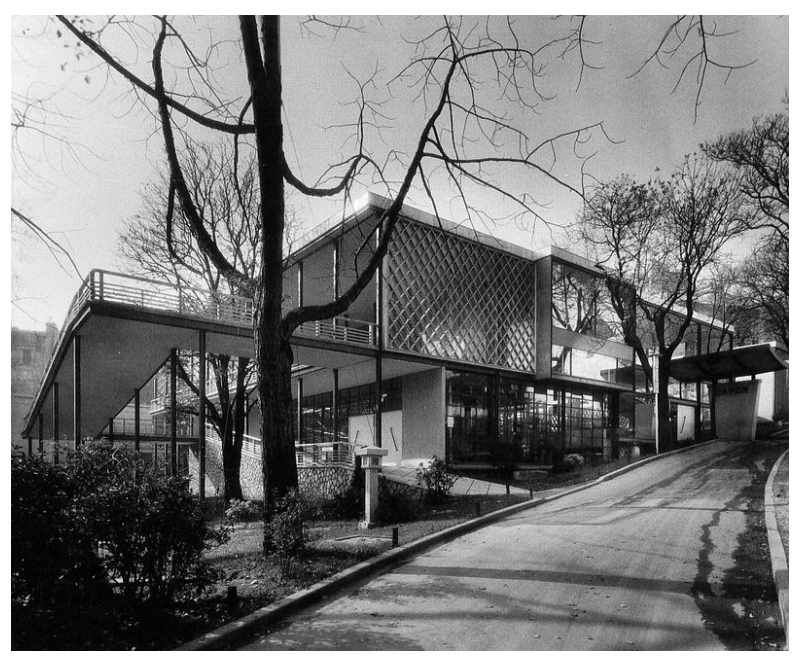

Fig. 5. Frank Lloyd Wright, Hotel Imperial de Tokio, 1916-22. Tomada del sitio https://commons.wikimedia.org/wiki/File:Imperial_Hotel_Wright_House.jpg

Por su parte, el arquitecto alemán Bruno Taut, representante de la Neues Bauen o Nueva Construcción en la Alemania de los años 20, fue otro de los arquitectos occidentales cuya obra se enmarca en torno a las nuevas corrientes impulsadas por

\footnotetext{
Hiтснсоск, Henry-Russell (1981): Arquitectura: Siglos XIX y XX. Madrid: Ediciones Cátedra, p. 470.

MeEch, Julia (2001): Frank Lloyd Wright and the Art of Japan. The architect's other passion. New York: Japan Society and Harry N. Abrams, Inc, Publishers, p. 38.

8 Osнima, Ken Tadashi (2010): International ..., op. cit p. 37. (Trad. de la autora.)
} 
el Movimiento Moderno que fueron más allá de la mera admiración por la estética japonesa y llegó a conocer in situ la "maravilla arquitectónica de Japón". Una serie de vicisitudes personales sobrevenidas tras la llegada al poder de los nazis, que ponen en su punto de mira al arquitecto debido a su ideología política claramente de izquierdas — el 1 de marzo de 1933, un día después de la quema del Reichtag, es advertido de su inminente detención y ese mismo día abandona Berlín junto a su compañera Erica Wittich - llevan a Bruno Taut a vivir en Japón durante tres años y medio. Su intención inicial es permanecer en el país por un periodo de tres meses, sin embargo, tras la denegación del visado para viajar a EEUU, se ve obligado a prolongar su estancia. Apenas unos días después de su llegada a Japón, coincidiendo con su cincuenta y tres cumpleaños, es invitado a visitar la Villa Imperial de Katsura, permiso que únicamente podía obtenerse por concesión especial de la corte japonesa. La Villa Katsura es en aquel tiempo un "conjunto que aunque citado en los libros de historia de la arquitectura, no aparece en ellos especialmente reseñado"10 según señala José Manuel García Roig en su obra Tres arquitectos alemanes. La profunda impresión que esta cumbre de la arquitectura tradicional japonesa causa en Taut le lleva a producir numerosos libros, artículos y conferencias dedicados a este estilo autóctono, pero también se anima a escribir sobre la situación de la arquitectura moderna en el país asiático, cuyo mejor ejemplo sería el texto Das japanische Haus und sein Leben. La declarada pasión de Bruno Taut por la arquitectura japonesa tradicional queda patente en muchos de esos escritos, opiniones como las que se encuentran en sus anotaciones tras su primera visita a Katsura: "Arquitectura reducida a la pura esencia. Emocionante, inocente como un niño. Satisfacción de una añoranza actual [...] belleza para la vista: el ojo se convierte en agente de lo espiritual. Así de bello se ofrece Japón a nuestra vista"11 u observaciones más objetivas como las del comienzo de su artículo La maravilla arquitectónica de Japón: "Para las personas instruidas de cualquier parte del mundo, Japón es el país del que han partido los estímulos más altamente significativos para el desarrollo del arte moderno" ${ }^{12}$. Se refiere aquí sobre todo al teatro y a la pintura, especialmente a las xilografías en color. A pesar de esta entusiasta actitud hacia la cultura y el arte japoneses en su vertiente tradicional, la arquitectura moderna del país, que como hemos indicado es también asunto de sus escritos en esta etapa japonesa, no le merece una opinión tan alta, como se extrae del artículo publicado en la revista L'Architecture d'Aujourd'hui, con el título Architecture nouvelle au Japon. Según leemos en la ya mencionada obra de José Manuel García Roig, el arquitecto alemán consideraba que en Japón no existía una auténtica arquitectura moderna debido a factores como la copia de modelos occidentales, la escasa independencia de la profesión o la falta de adaptación al clima japonés de las construcciones realizadas en la época.

Para rebatir los argumentos de Taut en contra de la existencia de una verdadera arquitectura moderna en Japón, podríamos mencionar las críticas hacia la imitación de modelos europeos de los sectores cercanos a la arquitectura oficialista por parte

\footnotetext{
9 Hacemos alusión directa aquí al artículo escrito por Bruno Taut Das architektonische Weltwunder Japans (La maravilla arquitectónica del Japón) publicado en la revista Nippon en enero de 1935 y cuyo texto íntegro es reproducido en el libro de García Roig, José Manuel (2004): Tres arquitectos alemanes: Bruno Taut, Hugo Häring, Martin Wagner. Valladolid: Universidad de Valladolid, pp. 106-111.

10 García Roig, José Manuel (2004): Tres arquitectos... op. cit., p. 98.

11 Ibid., p. 98.

12 Ibid., p. 106.
} 
de los miembros del grupo reformador Bunriha. El manifiesto del grupo creado en 1920, muy en la línea del carácter japonés, huye de la confrontación y del radicalismo propio de otros manifiestos artísticos occidentales de la época, sin embargo, se sitúa lejos de los que durante el proceso de modernización de Japón tras la Restauración Meiji consideraban que modernización equivalía a occidentalización. Según Oshima, el grupo de arquitectos Bunriha se declara equidistante frente a las posiciones antagonistas de las dos corrientes imperantes en la Universidad Imperial de Tokio. Por una parte, los partidarios de la arquitectura estructural, es decir, la que establece que la arquitectura no es arte sino funcionalidad, y por otra, la facción de los defensores del diseño frente a la técnica, que para arquitectos como Horiguchi y Yamada, más inclinados hacia el componente artístico de la arquitectura, resultaba sin embargo, inaceptablemente historicista. Es el mismo Oshima quien señala que la facción estructuralista recibe críticas también debido al creciente poder político que va obteniendo. Por tanto, la falta de independencia que denuncia Taut pudo darse en algunos círculos de la nueva arquitectura desarrollada en Japón, sin embargo, resulta poco convincente que toda la profesión estuviera condicionada por el sector oficial cercano al gobierno. Por último, si analizamos las obras de estos representantes de la modernidad, no parece que la arquitectura de los años veinte y treinta en Japón, o al menos el trabajo de los arquitectos mencionados aquí y algunos de sus colegas afines, diera la espalda a la conexión ancestral que en este país se da entre vida y naturaleza, por tanto entre arquitectura y los múltiples fenómenos relacionados con la climatología.

Hemos hablado de los trabajos de arquitectos occidentales en Japón y de la importancia de los viajes a Occidente en la asimilación de los principios de la nueva arquitectura por parte de los japoneses pero al igual que sus colegas occidentales de visita en el país asiático ¿tuvieron los japoneses posibilidades de trabajar en Occidente? Las fuentes parecen indicar que el papel de los arquitectos nipones se limitó al de alumno. En la obra de Tim Benton, El Estilo Internacional ${ }^{13}$ encontramos uno de los pocos ejemplos de proyecto diseñado y construido por un arquitecto japonés en Europa, el Pabellón Japonés de la Exposición Internacional de París de 1937 realizado por Sakakura Junzō [fig. 5], el cual había trabajado con Le Corbusier entre 1931 y 1936. En este edificio se combinan los valores estéticos de la construcción tradicional en madera con los principios del Estilo Internacional basados en el uso del hormigón y el acero.

\section{Conclusiones}

En el contexto de la relación y los intercambios entre Oriente y Occidente, la importancia que los viajes intercontinentales, realizados por profesionales de ambos mundos, tuvieron en el desarrollo de la arquitectura moderna japonesa cuenta con significativos ejemplos como hemos visto. Sin embargo, aunque la influencia occidental sobre el país nipón es considerada como evidente a través del estudio de las obras de los arquitectos que en Japón desarrollaron los principios que a lo largo de los años veinte y treinta cristalizaron en el denominado Estilo Internacional en Europa, los aspectos de la tradición japonesa que en sentido inverso fueron asimilados

13 Benton, Tim (1981): El Estilo Internacional 1. Madrid: Adir Editores. 
por "los padres" de la arquitectura moderna occidental e incorporados como principios completamente novedosos, no han sido ampliamente investigados. ¿En qué medida los principios de la arquitectura japonesa inspiraron a más de una generación de los considerados maestros occidentales? ¿Realmente los precursores de la revolución de la arquitectura de principios de siglo XX fueron impermeables a décadas de intercambios previos con Japón y modas procedentes del país asiático? Se trata de un capítulo del relato global sobre el Movimiento Moderno en el que los viajes realizados por los arquitectos japoneses tuvieron consecuencias directas sobre éstos, como la incorporación de lo ajeno y la integración de principios globales que impulsarían el desarrollo de la modernidad en Japón, sin duda alguna, pero que también cambiarían de forma irreversible la manera de ver la arquitectura de muchos de las más importantes y relevantes figuras occidentales. 